\title{
Movement disorder surgery, the essentials. Ed. Roy A. E. Bakay
}

\author{
Hardcover ISBN: 978-1-58890-397-6 Thieme
}

\author{
Jocelyne Bloch
}

Published online: 23 September 2009

(C) Springer-Verlag 2009

Movement Disorder Surgery, The Essentials is a hardcover book of around 250 pages that presents the current state of practice in the field.

Roy A. E. Bakey has invited 39 well-known experts to cover the topics, with each chapter ending with his personal comments.

The book is divided into 17 chapters, each reviewing a topic with description of the theoretical and practical aspects related to the field.

The first chapters cover the history, rationale, and mechanisms of action of movement disorder surgery, followed by chapters on patient selection, surgical procedures, alternative techniques, hardware, electrophysiology, and the most recent technical developments.

The chapters are clear, concise, with most of them citing over 100 references. Sometimes overlaps can be observed between chapters; however, the messages remain coherent. The tables and figures are didactic and help the reader to understand the concepts.

The reader will appreciate the constructive comments of the main editor at the end of each chapter. This gives the book a more critical view of the field.

The originality of the book is related to all the practical information provided to the reader. Indeed, the chapters on "Setting up a movement disorder surgery practice", "Preparation for movement disorder surgery", "Anesthesia for movement disorder surgery", Avoiding complications and correcting errors in movement disorder surgery", and "Deep brain stimulations programming", provide documentation on technical and practical aspects in addition to the costs of the procedures. These practical issues are generally not easily found in textbooks and the literature elsewhere; therefore, this book represents a valuable tool for any group that aims to setup a new movement disorder surgery unit.

Furthermore, this book not only covers the current and common way to do movement disorder surgery, but also with chapters like "Stereotactic surgery without microelectrode recording", "Implantation of multiple electrodes and robotic techniques", "Frameless functional stereotactic approaches" "Gamma Knife", and "The future of treatment for advanced Parkinson's disease", gives the opportunity to the reader to familiarize with more novel or controversial strategies, not generally performed by the majority of the functional neurosurgeons.

In summary, the authors have certainly accomplished the aim of giving an overview of movement disorder surgery. This book is directed primarily at practicing physicians who want to establish a new unit. They will certainly appreciate all the relevant practical issues which are discussed in a straightforward manner. Moreover, this manuscript offers a critical analysis of the state of the art in the field and opens perspectives in developments and alternative treatments.

J. Bloch $(\bowtie)$

Service de Neurochirurgie,

BH 8,

1005 Lausanne CHUV, Switzerland

e-mail: Jocelyne.Bloch@chuv.ch 